\title{
The HVP contribution to the anomalous magnetic moment of the muon
}

\section{T. H. Davies ${ }^{* \dagger}$}

School of Physics and Astronomy, University of Glasgow, UK

E-mail: christine.davieseglasgow.ac.uk

\section{Bipasha Chakraborty}

School of Physics and Astronomy, University of Glasgow, UK and Jefferson Lab, Virginia, USA

\section{P. G. De Oliveira}

School of Physics and Astronomy, University of Glasgow, UK

\section{J. Koponen}

School of Physics and Astronomy, University of Glasgow, UK

\section{G. P. Lepage}

LEPP, Cornell University, New York, USA

\section{HPQCD Collaboration}

\begin{abstract}
We report on the HPQCD calculation of the $\mathrm{u} / \mathrm{d}$ hadronic vacuum polarisation (HVP) contribution to the anomalous magnetic moment of the muon $\left(a_{\mu}\right)$ from full lattice QCD. This allows us to obtain a total leading order HVP contribution from $u, d, s, c$ and $b$ quarks. Our value is $666(6)(12) \times 10^{-10}$ including systematic uncertainties from quark-line disconnected pieces and QED and isospin effects. Our result implies a discrepancy between the experimental determination of $a_{\mu}$ and the Standard model of $3 \sigma$. We discuss prospects for improvements to this calculation underway with the MILC and Fermilab Lattice collaborations.
\end{abstract}

34th annual International Symposium on Lattice Field Theory

24-30 July 2016

University of Southampton, UK

\footnotetext{
${ }^{*}$ Speaker.

${ }^{\dagger}$ HPQCD Collaboration: www.physics.gla.ac.uk/HPQCD/
} 


\section{Introduction}

New particles can reveal their existence indirectly through tiny discrepancies in the properties of known particles from that expected in the Standard Model. The magnetic moment of the muon shows such a discrepancy, a tantalising 25 parts in $10^{10}$, with $3 \sigma$ significance. The magnetic moment, $\mu$, is given in terms of the spin, $S$, by:

$$
\vec{\mu}=g \frac{e}{2 m} \vec{S} ; \quad a_{\mu}=\frac{g-2}{2}
$$

The difference of $g$ from the naive value of 2 (all divided by 2) is called the anomalous magnetic moment, $a_{\mu}$. It is determined directly by measuring the spin precession as polarised muons circulate in a ring with a perpendicular magnetic field. Experiment E989 at Fermilab will start data-taking in 2017 and aims to reduce the experimental uncertainty by a factor of 4 . An improved theoretical uncertainty from the Standard Model is needed to match this. The largest uncertainty comes from the diagram containing a quark loop (see Figure 1): the Leading Order Hadronic Vacuum Polarisation (HVP) contribution. We give here the results of a calculation of this contribution from full lattice QCD.
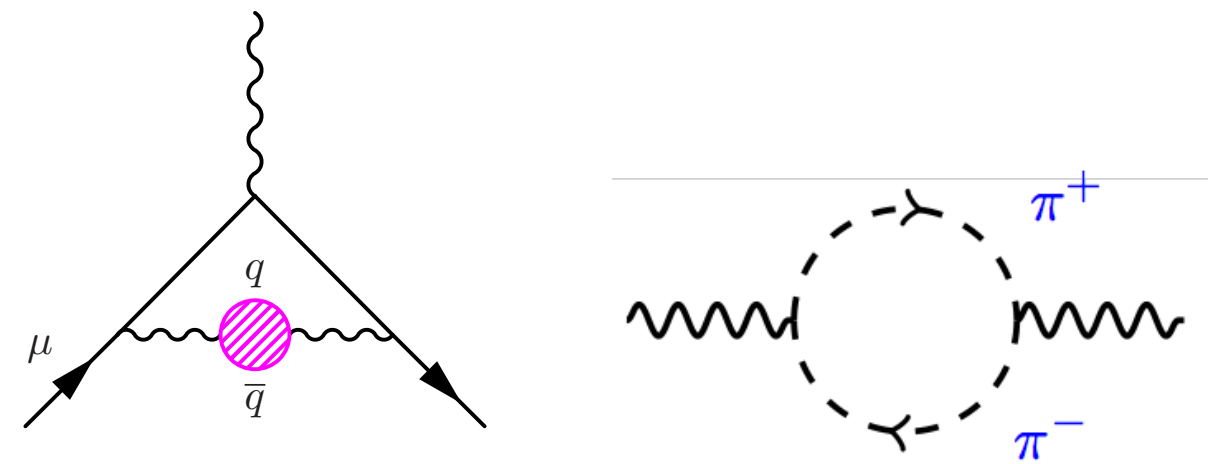

Figure 1: On the left, the $\alpha_{\mathrm{Q} E D}^{2}$ leading order HVP contribution to $a_{\mu}$ is represented as a strongly-interacting shaded blob inserted into the photon propagator that corrects the point-like photon-muon coupling at the top of the diagram. This figure shows the dominant connected contribution where a single $q \bar{q}$ pair is produced as a virtual intermediate state in the photon propagator. This is flavor diagonal. There is in addition a small quark-line disconnected contribution from cases where the original $q \bar{q}$ pair annihilate to gluons at an intermediate point and a new $q \bar{q}$ pair is generated from the gluons to once again annihilate to a photon. This process is not flavor-diagonal but is suppressed when summed over $u, d$ and $s$ quarks because the sum of their electric charges vanishes.

On the right, the leading order chiral perturbation theory (scalar QED) diagram for the HVP contribution, from $\gamma \rightarrow \pi^{+} \pi^{-}$. Chiral perturbation theory on its own does not provide a good description of the $u / d$ HVP contribution to $a_{\mu}$ since that contribution is dominated by the $\rho$. However, the $\gamma \rightarrow \pi \pi$ diagram above is sensitive to $m_{\pi}$ and the finite volume and so is important in studies of systematic uncertainties from those sources in lattice QCD calculations.

\section{Lattice Calculation}

We use MILC gluon field configurations that include $u, d, s$ and $c$ HISQ sea quarks (with $\left.m_{u}=m_{d}\right)$ at 3 lattice spacing values $(0.15,0.12$ and $0.09 \mathrm{fm}), 3$ u/d quark masses going down to 
the physical $u / d$ quark mass and 3 volumes (for one parameter set). This allows us to do the most realistic calculations of the HVP contribution to $a_{\mu}$ to date [1]. On these configurations we generate HISQ propagators and combine them to give the correlation function between two local spatial vector currents constructed of quark fields of a specfic flavor (normalised nonperturbatively), separated by a time interval, $t$. Taking $t$-moments of the correlation function allows us to reconstruct the renormalised vacuum polarisation function [2] and determine, by integrating over $k^{2}$, its contribution to $a_{\mu}$.

Ignoring quark-line disconnected contributions, the vacuum polarization separates into distinct contributions for each quark flavour, $\mathrm{f}$ :

$$
a_{\mu}^{\mathrm{HVP}, \mathrm{LO}}(\mathrm{f})=\frac{\alpha}{\pi} \int_{0}^{\infty} d k^{2} f\left(k^{2}\right)(4 \pi \alpha) \hat{\Pi}_{\mathrm{f}}\left(k^{2}\right)
$$

where $\alpha \equiv \alpha_{\mathrm{QED}}$ is the QED fine structure constant and $k$ is the (Euclidean) momentum carried by the virtual photons. $f\left(k^{2}\right)$ is a kinematic factor that diverges as $k^{2} \rightarrow 0$, where the renormalized vacuum polarization function, $\hat{\Pi}\left(k^{2}\right) \equiv \Pi\left(k^{2}\right)-\Pi(0)$, vanishes [3]. The resulting integrand is peaked around $k^{2} \approx m_{\mu}^{2}$. Note that, in this expression, we have absorbed into $\hat{\Pi}$ a factor of $Q_{\mathrm{f}}^{2}$, where $Q_{\mathrm{f}}$ is the electric charge of quark $\mathrm{f}$ in units of the proton's charge. This is a change to the convention that we used in [2].

Lattice QCD can be used to calculate the vacuum polarization function $\hat{\Pi}\left(k^{2}\right)$. In [2] we developed an accurate method using correlators that are routinely calculated for determining the hadron spectrum. We define $\hat{\Pi}\left(k^{2}\right)$ in terms of its Taylor expansion,

$$
\hat{\Pi}\left(k^{2}\right)=\sum_{j=1}^{\infty} k^{2 j} \Pi_{j}
$$

where the Taylor coefficients $\Pi_{j}$ are determined from time-moments $G_{2 j}$ of the vector currentcurrent correlator at zero spatial momentum:

$$
G_{2 j} \equiv \sum_{t} \sum_{\vec{x}} t^{2 j} Z_{V}^{2}\left\langle j^{i}(\vec{x}, t) j^{i}(0)\right\rangle ; \quad Q_{\mathrm{f}}^{2} G_{2 j}=\left.(-1)^{j} \frac{\partial^{2 j}}{\partial k^{2 j}} k^{2} \hat{\Pi}\left(k^{2}\right)\right|_{k^{2}=0}=(-1)^{j}(2 j) ! \Pi_{j-1} .
$$

Here $Z_{V}$ renormalizes the lattice vector current, and

$$
t \in(0,1,2, \ldots T / 2-1,0,-T / 2+1, \ldots,-2,-1) .
$$

We replace the Taylor series by its $[n, n]$ and $[n, n-1]$ Padé approximants to perform the integral in Eq. (2.1) numerically. The approximants provide an accurate approximation for both the low and high $k^{2}$ regions in the integral, and results converge to better than $1 \%$ by $n=2$ [2].

For $s, c$, and $b$ quarks direct computation of the moments from the lattice correlators is very accurate $[2,4,5]$. For the $u / d$ case [1] we reduce statistical errors by calculating a $2 \times 2$ matrix of local and smeared correlators and using fitted results for the local correlator at large $t$ in the moment caclulation, rather than the data.

The $\rho$ pole dominates the $u / d$ HVP calculation, giving $70 \%$ of the result. Properties of the $\rho$, such as its mass and decay constant, obtained from our correlators at large $t$ can be compared to experiment (see Figure 2), where we see good agreement at physical $u / d$ quark masses. Note 

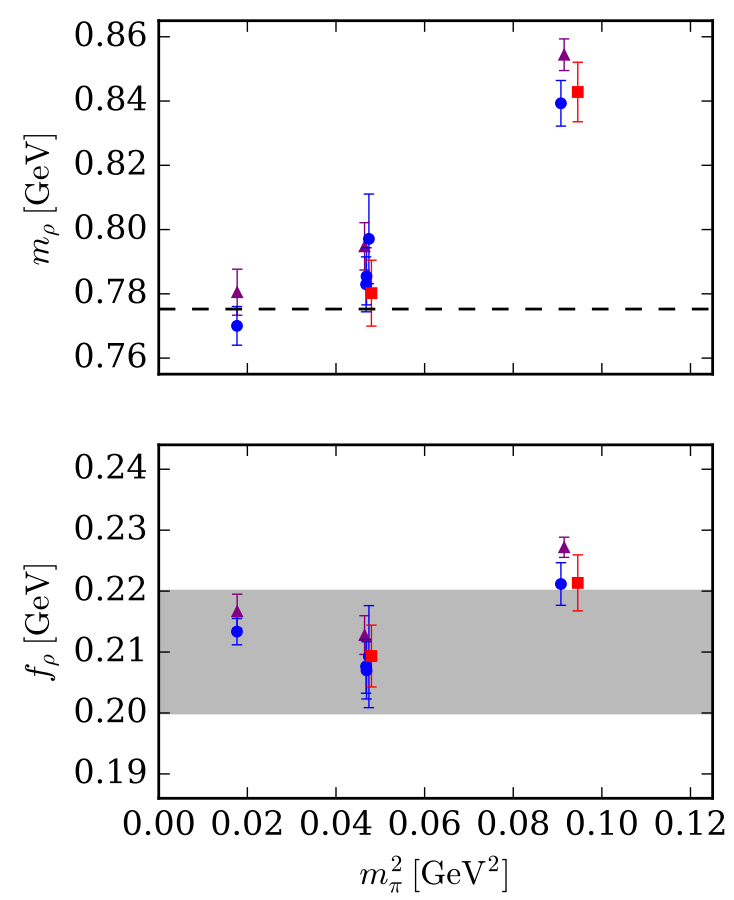

Figure 2: Results for the $\rho$ meson mass (upper plot) and decay constant (lower plot) as a function of $u / d$ quark mass given by $m_{\pi}^{2}$. These are obtained from large-time fits to the vector correlators used for our determination of the $u / d$ HVP contribution to $a_{\mu}$. Experimental results are given as a dashed line for the mass and a grey band for the decay constant, where the uncertainty comes from the definition of the decay constant for a particle such as the $\rho$, which has a large width.

that, although our $\pi$ masses are light enough and our volumes large enough for $\rho$ decay, our correlators do not give the ground-state $\pi \pi$ energy (corresponding to each $\pi$ having one unit of spatial momentum) at large $t$. This is because the local vector-vector correlator, which is the correct one to calculate for $a_{\mu}$, is not designed to have good overlap with $\pi-\pi$ states.

At smaller $t$, and in the $t$-moments, additional states beyond the $\rho$ contribute, including excited $\rho$ states and $\pi \pi$ states with back-to-back $\pi$ with the full range of lattice spatial momenta. The leading $\pi \pi$ terms are readily calculated in scalar QED (see Figure 1, right) and the continuum integrals and lattice sums over momenta performed for different $m_{\pi}$ and different lattice volumes. This calculation is very accurate since the only parameters are $m_{\pi}$ and $e_{\pi}$. The $\pi \pi$ contribution is the piece most sensitive to the volume and the $\pi$ mass and it is therefore important to study this to understand lattice QCD systematic uncertainties. A specific staggered quark issue relevant to our calculation is that different tastes of $\pi$ meson have masses that differ at $\mathscr{O}\left(a^{2}\right)$ and a sum over tastes must be done [1]. We use this calculation to determine corrections to our lattice QCD results for the $\pi \pi$ contribution; not surprisingly these corrections are most significant for physical $m_{\pi}$. We also estimate corrections to this analysis by coupling in an explicit $\rho$ field to the effective theory with parameters $m_{\rho}, f_{\rho}, f_{\rho \pi \pi}$ that are also well-known. Our correction from finite-volume/staggered quark effects in $\pi \pi$ is $7.0(7) \%$ at physical $u / d$ masses. In addition, sensitivity to the $u / d$ quark mass in our results is almost eliminated by rescaling the moments (with $\pi \pi$ removed) by appropriate 
powers of the experimental $\rho$ mass, modifying a method in [6]. This affects results at unphysically heavy $u / d$ masses.

Figure 3 shows corrected and raw data as a function of the $u / d$ quark mass. Note how the corrected data have reduced volume, $m_{u / d}$ and $a$-dependence. A simple fit gives a physical value for the connected $u / d$ HVP contribution of 598(6)(8)x10 $10^{-10}$ where 6 is the fit/statistical uncertainty and 8 is the systematic uncertainty from missing QED and isospin effects. The probability density function for the result is shown in Figure 3.
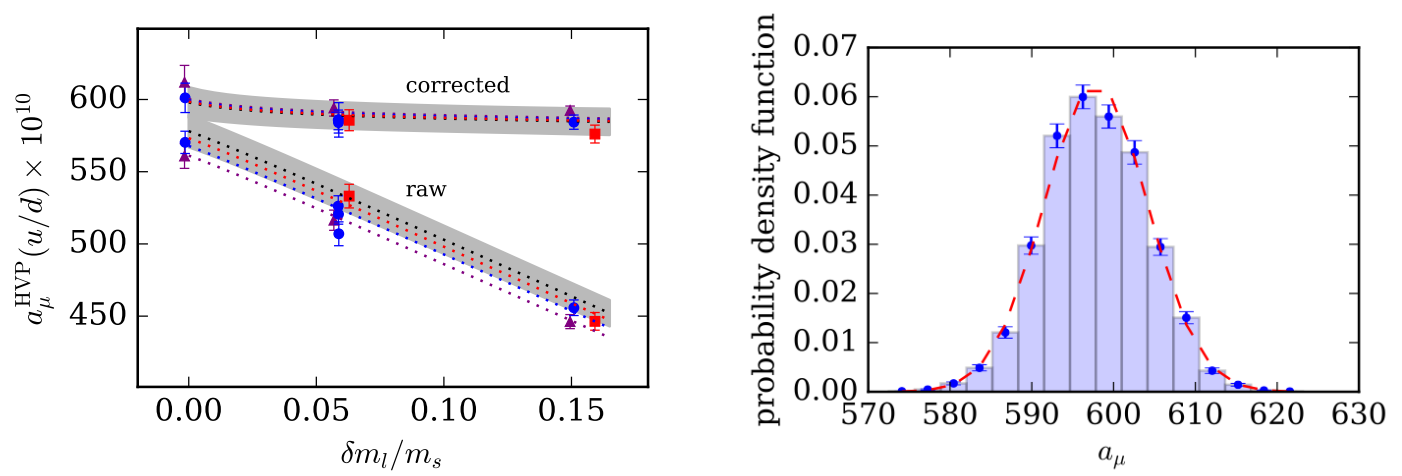

Figure 3: On the left: our results for the connected $u / d$ contribution to $a_{\mu}^{H V P, L O}$. This is plotted against the $u / d$ quark mass, expressed as its deviation from the physical value in units of the tuned $s$ qaurk mass. The lower curve shows our raw data; the upper curve includes corrections to the $\pi \pi$ contribution and a rescaling of moments away from the physical point by powers of the $\rho$ mass. Different symbols denote results at different values of the lattice spacing, $a$. The dotted lines show fitted results at each value of $a$ with the black dotted line representing the continuum limit. The grey bands give $\pm 1 \sigma$ about that result.

On the right: the probability distribution function for our final result for the $u / d$ connected contribution to $a_{\mu}^{H V P, L O}$ from a Bayesian analysis of our fit. The red line is the Gaussian approximation to the distribution, which agrees well.

Combining the $u / d$ result with our earlier values for the contribution from $s$ quarks [2], $c$ quarks [2,4] and $b$ quarks [5] gives a total connected HVP,LO result from adding

$$
\left.a_{\mu}^{\text {HVP,LO }}\right|_{\text {conn. }} \times 10^{10}= \begin{cases}598(11) & \text { from } u / d \text { quarks } \\ 53.4(6) & \text { from } s \text { quarks } \\ 14.4(4) & \text { from } c \text { quarks } \\ 0.27(4) & \text { from } b \text { quarks }\end{cases}
$$

to obtain

$$
a_{\mu}^{H V P, L O}=666(6)(12) \times 10^{-10} .
$$

This now includes a $1.5 \%$ systematic uncertainty for quark-line disconnected diagrams obtained from an analysis of Hadspec collaboration correlators [7]. These have a clear signal for disconnected contributions for all light flavour combinations, albeit at unphysically heavy $u / d$ quark masses. Simple phenomenological arguments based on the properties of $\rho$ and $\omega$ mesons also lead to the conclusion that the quark-line disconnected contribution is $\mathscr{O}(1 \%)$. A calculation with domain-wall quarks now gives a non-zero result in agreement with this expectation [8]. 
We compare lattice results to determinations that use the experimental cross-section for $e^{+} e^{-} \rightarrow$ hadrons in Figure 3. The green point shows the value expected if there were no new physics (i.e. this point is the value of experiment - [sum of Standard Model values for QED, EW, HVP,Higher Order and hadronic light-by-light contributions $]=720(7) \times 10^{-10}$ ).

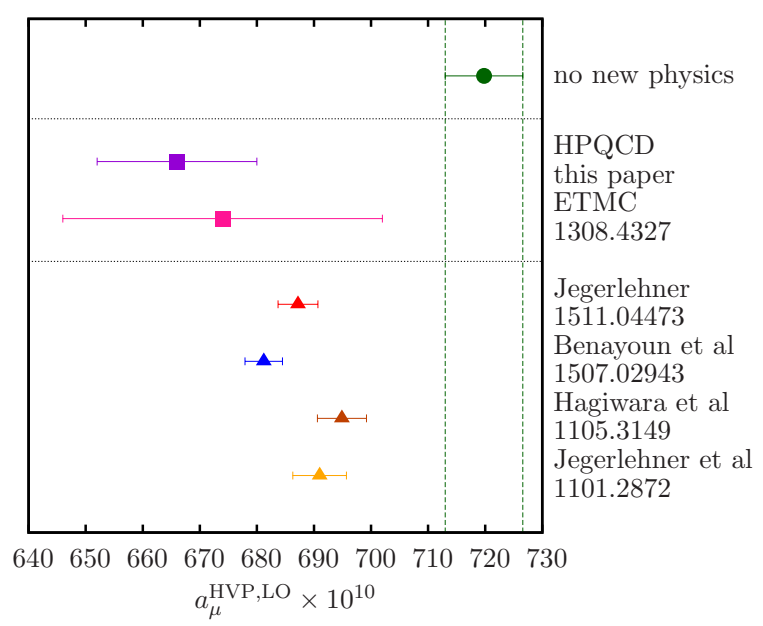

Figure 4: Our final result for $a_{\mu}^{H V P, L O}$ compared to an earlier value (including $u / d, s$ and $c$ quarks) from the ETM collaboration [6] and to recent results using experimental cross-section information from $e^{+} e^{-} \rightarrow$ hadrons $[9,10,11,12]$. The green filled circle denotes the value expected for the HVP contribution working back from the experimental result in the absence of new physics. Our value differs from this by $3 \sigma$.

\section{Conclusions}

Lattice QCD calculations of the HVP contribution to $a_{\mu}$ are making fast progress. Our result is the most accurate to date and shows a $3 \sigma$ discrepancy with experiment. Ongoing work with the MILC and Fermilab Lattice collaborations is improving results at the physical value of $m_{u / d}$ by increasing statistics on the lattices used here and also going to finer lattices where staggered quark taste effects will be reduced. We are adding analysis of QED and isospin effects along with improved analysis of disconnected contributions.

\section{References}

[1] B. Chakraborty et al, HPQCD collaboration, arXiv:1601.03071.

[2] B. Chakraborty et al, HPQCD collaboration, Phys. Rev. D89:114501 (2014), arXiv:1403.1778.

[3] T. Blum, Phys. Rev. Lett.91:052001 (2003), arXiv:hep-lat/0212018.

[4] G. Donald et al, HPQCD Collaboration, Phys. Rev. D86:094501 (2012), arXiv:1208.2855.

[5] B. Colquhoun et al, HPQCD Collaboration, Phys. Rev. D91:074514 (2015), arXiv:1408.5768.

[6] F. Burger et al, JHEP 1402:099 (2014), arXiv:1308.4327.

[7] B. Chakraborty et al, Phys. Rev. D93:074509 (2016), arXiv:1512.03270.

[8] T. Blum et al, Phys. Rev. Lett.116:232002 (2016), arXiv:1512.09054. 
[9] K. Hagiwara et al, J. Phys. G38:085003 (2011), arXiv:1105.3149.

[10] F. Jegerlehner and R. Szafron, Eur. Phys. J. C71:1632 (2011), arXiv:1101.2872.

[11] M. Benayoun et al, Eur. Phys. J. C75:613 (2015), arXiv:1507.02943.

[12] F. Jegerlehner, EPJ Web Conf. 118:01016 (2016), arXiv:1511.04473. 